\title{
USING FACTORIZATION IN ANALYZING D-BMAP/G/1 QUEUES
}

HO WOO LEE, SE WON LEE, AND JONGWOO JEON

Received 12 March 2004 and in revised form 8 September 2004

We demonstrate how one can use the factorization property to derive the queue-length distributions of the discrete-time BMAP/G/1 queues with complex operational behavior during the idle period. The procedure demonstrated in this paper can be applied to the analysis of many other discrete-time BMAP/G/1 queues with more behavioral complexities.

\section{Introduction}

The discrete-time batch Markovian arrival process (D-BMAP) was first defined in [2]. The D-BMAP can represent a variety of arrival processes which include, as special cases, the Bernoulli arrival process, the Markov-modulated Bernoulli process (MMBP), the discrete-time Markovian arrival process (D-MAP), and their superpositions. It is the discrete-time version of the versatile Markovian point process introduced by Neuts [28], the $N$-process of Ramaswami [31], and the batch Markovian arrival process of Lucantoni $[25,26]$.

The objective of this paper is to demonstrate how one can apply the factorization property to the derivation of the queue-length distributions of the D-BMAP/G/1 queues with complex operational behavior during the idle period. To demonstrate how this new approach works, we are going to analyze the D-BMAP/G/1 queueing system under a double threshold policy and a setup time, which becomes the basic model for many production systems. The approach in this paper is simpler than the conventional matrix analytic method (MAM) and the supplementary variable technique.

The MAM was pioneered by Neuts [29]. It starts with the analysis of the imbedded Markov renewal process at departure epochs. This method is cumbersome, especially in a system with a high degree of behavioral complexities during the idle period, in that it involves manipulating the vast amount of matrices without knowing the practical meaning of the resulting matrices. Works based on MAM are many. Blondia and Casals [2] modeled a digital video communication system by D-BMAP. Hashida et al. [7] analyzed the system with switched batch Bernoulli process (SBBP) with and without priorities. Ishizaki et al. [10] analyzed the SBBP/G/1 system in which the staying time of the underlying 
Markov chain (UMC) follows a general distribution. Alfa and Neuts [1] analyzed the vehicular traffic system by D-MAP. Other studies on queueing systems with D-BMAP or D-MAP can be found in $[5,6,11,12,16,30,32,33,34,36]$, to name a few. For a detailed study concerning applications to communication models, see Bruneel and Kim [3]. Computational algorithms for BMAP queues can be found in $[17,25,26]$. For continuous time analysis of BMAP queues, see Lucantoni [25, 26, 27] and Kasahara et al. [13].

The supplementary variable technique for D-BMAP/G/1 queues was used by Lee et al. $[23,24]$. It starts with setting up the system equations by using the forward recurrence (or backward recurrence) times of involved random variables as supplementary variables. But even with its obvious advantages in obtaining more diverse and meaningful results, this approach requires time-consuming effort in handling the system equations. For the analysis of continuous-time MAP/G/1 queues by supplementary variable technique, see Lee et al. [19] and the references therein.

In this paper, we first introduce the factorization property for D-BMAP/G/1 queues with generalized vacations. Then, we use this property to demonstrate how one can efficiently and effectively derive the queue-length distributions of some complicated DBMAP/G/1 queueing systems by avoiding all the classical standard procedures. For the application of continuous-time factorization principle to complex BMAP/G/1 queues, readers are referred to Lee et al. [21, 22].

\section{The factorization property}

Chang et al. [4] proved that for the D-BMAP/G/1 queues with generalized vacations (based on late-arrival model (see Takagi [35])), the following factorization properties hold:

$$
\begin{aligned}
& \mathbf{Y}(z)=\mathbf{p}_{\text {idle }}(z) \chi_{Y}(z), \\
& \mathbf{X}(z)=\operatorname{p}_{\text {idle }}(z) \chi_{X}(z),
\end{aligned}
$$

where

$$
\begin{aligned}
& \chi_{Y}(z)=(1-\rho)(z-1) \mathbf{A}(z)[z \mathbf{I}-\mathbf{A}(z)]^{-1}, \\
& \chi_{X}(z)=\frac{1}{\lambda}(1-\rho)[\mathbf{D}(z)-\mathbf{I}] \mathbf{A}(z)[z \mathbf{I}-\mathbf{A}(z)]^{-1} .
\end{aligned}
$$

In (2.1), $\mathbf{Y}(z)$ is the vector generating function (GF) of the queue length at an arbitrary slot boundary and $\mathbf{X}(z)$ is the vector GF of the queue length just after an arbitrary departure. $\mathbf{p}_{\text {idle }}(z)$ is the vector GF of the queue length at an arbitrary slot boundary during an idle period. In (2.2), $\mathbf{D}(z)=\sum_{n=0}^{\infty} \mathbf{D}_{n} z^{n}$, where $\mathbf{D}_{n}$ is the matrix of the arrival probabilities for a group of size $n$ during a slot. $\lambda=\pi \sum_{n=1}^{\infty} n \mathbf{D}_{n} \mathbf{e}$ is then the mean arrival rate per slot where $\pi$ is the stationary vector of the UMC that satisfies

$$
\pi=\pi \mathrm{D}, \quad \pi \mathrm{e}=1,
$$




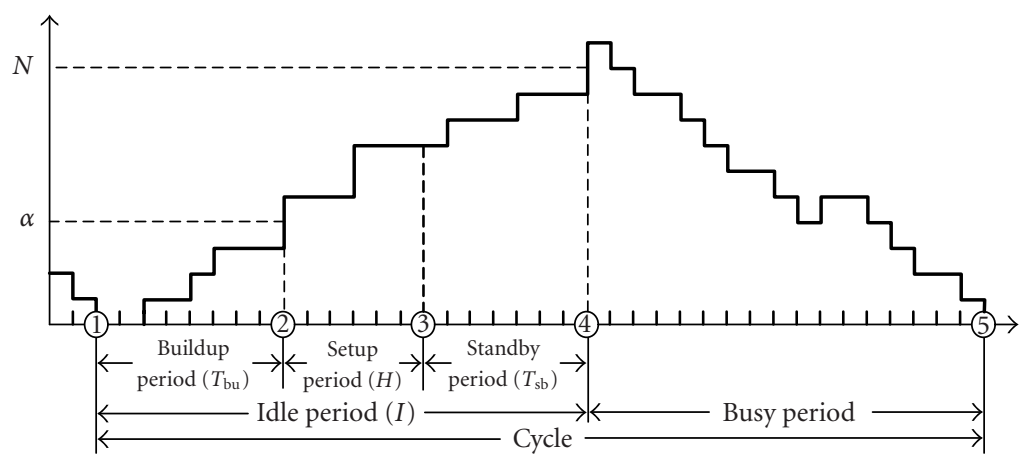

Figure 3.1. The system.

in which $\mathbf{D}=\left.\mathbf{D}(z)\right|_{z=1}=\sum_{n=0}^{\infty} \mathbf{D}_{n}$ and $\mathbf{e}$ is the $(m \times 1)$ vector of 1's. $\mathbf{A}(z)=\sum_{k=1}^{\infty} s_{k}[\mathbf{D}(z)]^{k}$ is the matrix GF of the number of customers that arrive during a service time in which $s_{k}$ is the probability that a service time is of length $k$ slots.

From (2.1), we note that the following relationship holds between $\mathbf{X}(z)$ and $\mathbf{Y}(z)$ :

$$
\mathbf{Y}(z)[\mathbf{D}(z)-\mathbf{I}]=\lambda(z-1) \mathbf{X}(z)
$$

which confirms Kim et al. [15].

Equation (2.1) implies that if one wants to derive the GFs $\mathbf{Y}(z)$ and $\mathbf{X}(z)$, all they need to do is derive the vector GF $\mathbf{p}_{\text {idle }}(z)$ of the queue length at an arbitrary slot boundary during an idle period.

\section{The system}

In this paper, we deal with the queueing system with the following specifications (Figure $3.1)$.

(1) Customers arrive according to the D-BMAP in which the UMC is governed by parameter matrices $\left\{\mathbf{D}_{0}, \mathbf{D}_{1}, \mathbf{D}_{2}, \ldots\right\}$. We assume that the UMC has $m$ phases.

(2) If there are no customers to serve (point (1) in Figure 3.1), the server waits until the queue length reaches or exceeds the first threshold $\alpha$ (buildup period).

(3) At the end of the buildup period, the server starts a setup period which takes a random time $H$ with $h_{k}=\operatorname{Pr}(H=K)$. At the end of the setup period,

(i) if the queue length is less than the second threshold $N$, the server waits until queue length reaches or exceeds $N$ (standby period); or

(ii) if the queue length is greater than or equal to $N$, the server begins to serve the customers (busy period).

(4) The service time $S$ is a random variable with $s_{k}=\operatorname{Pr}(S=K)$.

(5) The service times and setup times are independent of the arrival process and the phases of the UMC.

The objective of this study is to analyze the above queueing system by applying the factorization property of the D-BMAP/G/1 queue with generalized vacations directly to 
the vector generating functions of the queue lengths. We also derive the mean queue length.

This queueing system can be used to model the production system in which the setup cost is extremely high. Lee and Park [20] showed that the double threshold $(\alpha, N)$-policy is better than the single threshold $N$-policy when the setup cost is extremely high compared to the work-in-process (WIP) holding cost. We note that Lee et al. [19] applied the factorization to the analysis of WIP of a production system.

If $\alpha=N$, we have the usual $N$-policy queue with a setup. $N$-policy queue was first studied by Yadin and Naor [37]. For other works on $N$-policy queues, see, for example, $[8,9,14,18]$.

\section{The analysis}

From (2.1), we only need to derive the vector GF $\mathbf{p}_{\text {idle }}(z)$ of the queue length at an arbitrary slot boundary during an idle period.

We define $p_{\mathrm{bu}}, p_{\mathrm{su}}$, and $p_{\mathrm{sb}}$ as the probabilities that the server is in the buildup period, setup period, and standby period, respectively, under the condition that the server is idle. If we define $\mathbf{p}_{\mathrm{bu}}(z), \mathbf{p}_{\mathrm{su}}(z)$, and $\mathbf{p}_{\mathrm{sb}}(z)$ as the conditional vector GF of the queue length at an arbitrary point within the buildup, setup, and standby periods, respectively, we get

$$
\mathbf{p}_{\text {idle }}(z)=p_{\text {bu }} \mathbf{p}_{\mathrm{bu}}(z)+p_{\mathrm{su}} \mathbf{p}_{\mathrm{su}}(z)+p_{\mathrm{sb}} \mathbf{p}_{\mathrm{sb}}(z)
$$

To obtain the probabilities $p_{\mathrm{bu}}, p_{\mathrm{su}}$, and $p_{\mathrm{sb}}$, we need the mean lengths $E(I)$ of an idle period, $E\left(T_{\mathrm{bu}}\right)$ of a buildup period, and $E\left(T_{\mathrm{sb}}\right)$ of a standby period. In the sequel, we will use $(\mathbf{F})_{i j}$ to denote the $(i, j)$-element of the matrix $\mathbf{F}$.

We define the probabilities $\left(\Psi_{k}^{\mathrm{bu}}\right)_{i j}$ and $\left(\boldsymbol{\Psi}_{k}^{\mathrm{sb}}\right)_{i j}$ as follows:

(a) $\left(\Psi_{k}^{b u}\right)_{i j}$ denotes $\operatorname{Pr}$ (the buildup process ever visits level $k$ and the phase of UMC is $j$ just after the visit I UMC phase is $i$ at (1) of Figure 3.1);

(b) $\left(\Psi_{k}^{\mathrm{sb}}\right)_{i j}$ denotes $\operatorname{Pr}$ (the standby process ever visits level $k$ and the phase of UMC is $j$ just after the visit I UMC phase is $i$ at (1) of Figure 3.1).

Let $\boldsymbol{\kappa}=\left(\kappa_{1}, \kappa_{2}, \ldots, \kappa_{m}\right)$ be the probability vector of the UMC phase at (1). Noting that the $(i, j)$-element of the matrix $\left(\mathbf{I}-\mathbf{D}_{0}\right)^{-1}$ is the mean time the UMC stays in phase $j$ until the next arrival given the current phase is in $i$, we have

$$
\begin{aligned}
& E\left(T_{\mathrm{bu}}\right)=\boldsymbol{\kappa} \sum_{k=0}^{\alpha-1} \boldsymbol{\Psi}_{k}^{\mathrm{bu}}\left(\mathbf{I}-\mathbf{D}_{0}\right)^{-1} \mathbf{e}, \\
& E\left(T_{\mathrm{sb}}\right)=\boldsymbol{\kappa} \sum_{k=\alpha}^{N-1} \boldsymbol{\Psi}_{k}^{\mathrm{sb}}\left(\mathbf{I}-\mathbf{D}_{0}\right)^{-1} \mathbf{e} .
\end{aligned}
$$

Then, we get

$$
E(I)=\boldsymbol{\kappa}\left[\sum_{k=0}^{\alpha-1} \boldsymbol{\Psi}_{k}^{\mathrm{bu}}\left(\mathbf{I}-\mathbf{D}_{0}\right)^{-1}+E(H) \mathbf{I}+\sum_{k=\alpha}^{N-1} \boldsymbol{\Psi}_{k}^{\mathrm{sb}}\left(\mathbf{I}-\mathbf{D}_{0}\right)^{-1}\right] \mathbf{e} .
$$


Thus, we have

$$
\begin{aligned}
& p_{\mathrm{bu}}=\frac{E\left(T_{\mathrm{bu}}\right)}{E(I)}=\frac{\boldsymbol{\kappa} \sum_{k=0}^{\alpha-1} \boldsymbol{\Psi}_{k}^{\mathrm{bu}}\left(\mathbf{I}-\mathbf{D}_{0}\right)^{-1} \mathbf{e}}{E(I)}, \\
& p_{\mathrm{su}}=\frac{E(H)}{E(I)}, \\
& p_{\mathrm{sb}}=\frac{E\left(T_{\mathrm{sb}}\right)}{E(I)}=\frac{\boldsymbol{\kappa} \sum_{k=\alpha}^{N-1} \boldsymbol{\Psi}_{k}^{\mathrm{sb}}\left(\mathbf{I}-\mathbf{D}_{0}\right)^{-1} \mathbf{e}}{E(I)} .
\end{aligned}
$$

Noting that the $j$ th element of the vector $\boldsymbol{\kappa} \sum_{k=0}^{\alpha-1} \Psi_{k}^{\mathrm{bu}}\left(\mathbf{I}-\mathbf{D}_{0}\right)^{-1} / E\left(T_{\mathrm{bu}}\right)$ is the probability that the queue length is $k$ and the UMC phase is $j$ under the condition that the server is in the buildup period, we get

$$
\mathbf{p}_{\mathrm{bu}}(z)=\frac{\boldsymbol{\kappa} \sum_{k=0}^{\alpha-1} \boldsymbol{\Psi}_{k}^{\mathrm{bu}}\left(\mathbf{I}-\mathbf{D}_{0}\right)^{-1} z^{k}}{E\left(T_{\mathrm{bu}}\right)}
$$

which yields, from (4.4a),

$$
p_{\mathrm{bu}} \mathbf{p}_{\mathrm{bu}}(z)=\frac{\boldsymbol{\kappa} \sum_{k=0}^{\alpha-1} \boldsymbol{\Psi}_{k}^{\mathrm{bu}}\left(\mathbf{I}-\mathbf{D}_{0}\right)^{-1} z^{k}}{E(I)}
$$

Analogously, we get

$$
p_{\mathrm{sb}} \mathbf{p}_{\mathrm{sb}}(z)=\frac{\boldsymbol{\kappa} \sum_{k=\alpha}^{N-1} \boldsymbol{\Psi}_{k}^{\mathrm{sb}}\left(\mathbf{I}-\mathbf{D}_{0}\right)^{-1} z^{k}}{E(I)}
$$

To obtain $\mathbf{p}_{\mathrm{su}}(z)$, let $\mathbf{H}_{\alpha}^{-}(z)$ be the matrix GF of the queue length at the start of the setup period (point (2) of Figure 3.1). Then, we have

$$
\mathbf{p}_{\mathrm{su}}(z)=\boldsymbol{\kappa} \mathbf{H}_{\alpha}^{-}(z) \mathbf{H}_{e}(z)
$$

where $\mathbf{H}_{e}(z)$ is the matrix GF of the number of customers that arrive during the elapsed time, which is given by

$$
\mathbf{H}_{e}(z)=\sum_{k=1}^{\infty} \sum_{i=0}^{k-1}[\mathbf{D}(z)]^{i} \frac{1}{k} \cdot \frac{k h_{k}}{E(H)}=\frac{[\mathbf{H}(z)-\mathbf{I}][\mathbf{D}(z)-\mathbf{I}]^{-1}}{E(H)}
$$




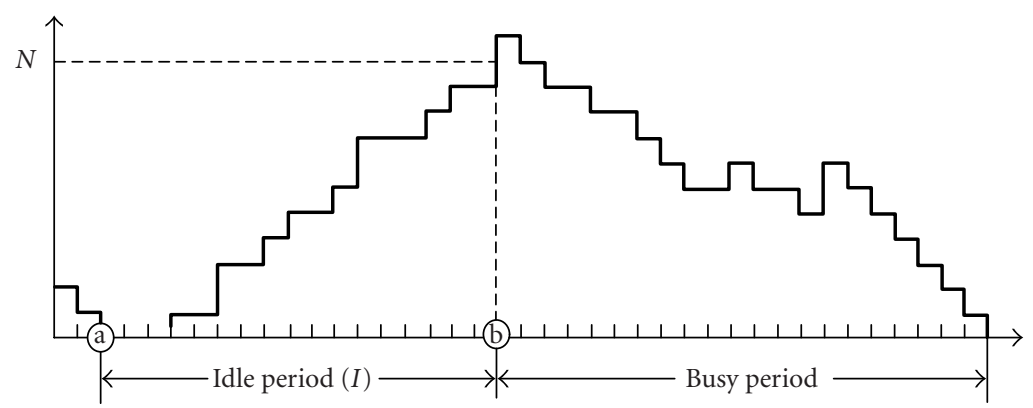

Figure 4.1. The D-BMAP/G/1 queue with $N$-poilcy.

in which $\mathbf{H}(z)=\sum_{k=1}^{\infty} h_{k}[\mathbf{D}(z)]^{k}$. Using (4.6a)-(4.8) in (4.1), we get

$$
\begin{aligned}
\mathbf{p}_{\text {idle }}(z)= & \frac{\boldsymbol{\kappa} \sum_{k=0}^{\alpha-1} \boldsymbol{\Psi}_{k}^{\mathrm{bu}}\left(\mathbf{I}-\mathbf{D}_{0}\right)^{-1} z^{k}}{E(I)}+\frac{E(H)}{E(I)} \boldsymbol{\kappa} \mathbf{H}_{\alpha}^{-}(z) \mathbf{H}_{e}(z) \\
& +\frac{\boldsymbol{\kappa} \sum_{k=\alpha}^{N-1} \boldsymbol{\Psi}_{k}^{\mathrm{sb}}\left(\mathbf{I}-\mathbf{D}_{0}\right)^{-1} z^{k}}{E(I)}
\end{aligned}
$$

Now, we need a scheme to compute the probabilities $\Psi_{k}^{\text {bu }}(0 \leq k \leq \alpha-1), \Psi_{k}^{\mathrm{sb}}(\alpha \leq k \leq$ $N-1$ ), and $\boldsymbol{\kappa}$. To this end, we observe that the behavior of the queueing process during the buildup period is exactly the same as that of the usual D-BMAP/G/1 system with simple $N$-policy (i.e., without setup time) where $N$ is replaced by $\alpha$. Figure 4.1 shows a sample path for the D-BMAP/G/1 queue with the simple $N$-policy.

Lemma 4.1 (D-BMAP/G/1/N-policy queue). Let $\left(\mathbf{D}_{n}^{*}\right)_{i j}$ be the probability that the idle period process of the D-BMAP/G/1/N-policy queue ever visits level $n$ and the UMC phase just after the visit is $j$ given that the UMC phase is $i$ at (a) of Figure 4.1. Then,

$$
\mathbf{D}_{0}^{*}=\mathbf{I}, \quad \mathbf{D}_{n}^{*}=\sum_{k=0}^{n-1} \mathbf{D}_{k}^{*}\left(\mathbf{I}-\mathbf{D}_{0}\right)^{-1} \mathbf{D}_{n-k} \quad(1 \leq n \leq N-1) .
$$

Proof. $\mathbf{D}_{0}^{*}=\mathbf{I}$ is obvious. Noting that $\left(\mathbf{I}-\mathbf{D}_{0}\right)^{-1} \mathbf{D}_{n}$ is the phase transition probability by the arrival of a group of size $n$, the proof is complete by conditioning on the level $k$ visited prior to level $n$.

Now, returning to our system, we get the following theorem without proof.

Theorem 4.2 .

$$
\Psi_{k}^{\mathrm{bu}}=\mathbf{D}_{k}^{*} \quad(0 \leq k \leq \alpha-1) .
$$

Now, computation of $\Psi_{k}^{\mathrm{sb}}(\alpha \leq k \leq N-1)$ is in order. We first note that $\boldsymbol{\Psi}_{k}^{\mathrm{sb}}$ depends on both the queue length at the start of the setup period and the number of customers that arrive during the setup time. Noting that the queue length at the start of the setup 
time is exactly equal to the queue length in the simple D-BMAP/G/1/N-policy queue where $N$ is replaced by $\alpha$, we present the following lemma.

LEMma 4.3 (BMAP/G/1/N-policy queue). Let $\left(\mathbf{Q}_{n}^{N}\right)_{i j}(n \geq N)$ be the probability that the queue length is $n$ and the UMC phase is $j$ at (b) of Figure 4.1 given that the UMC phase is in $i$ at (a). Then, there exists a recursion

$$
\mathbf{Q}_{n}^{N}=\left(\mathbf{I}-\mathbf{D}_{0}\right)^{-1} \mathbf{D}_{n}+\sum_{j=1}^{N-1}\left(\mathbf{I}-\mathbf{D}_{0}\right)^{-1} \mathbf{D}_{j} \mathbf{Q}_{n-j}^{N-j}
$$

and the matrix $G F \mathbf{Q}_{N}(z)$ of $\mathbf{Q}_{n}^{N}$ becomes

$$
\mathbf{Q}_{N}(z)=\sum_{n=N}^{\infty} z^{n} \mathbf{Q}_{n}^{N}=\left[\sum_{n=0}^{N-1} \mathbf{D}_{n}^{*}\left(\mathbf{I}-\mathbf{D}_{0}\right)^{-1} z^{n}\right]\left[\mathbf{D}(z)-\mathbf{D}_{0}\right]-\sum_{n=1}^{N-1} \mathbf{D}_{n}^{*} z^{n}
$$

Proof. Equation (4.12a) can be obtained by conditioning on the size of the first arrival group; (4.12b) can be obtained by mathematical induction.

We define the following notations:

(i) $\left(\mathbf{H}_{k(\alpha)}^{-}\right)_{i j}$ denotes the joint probability that the queue length is $k$ and the UMC phase is $j$ at (2) of Figure 3.1 given that the UMC phase is $i$ at (1),

(ii) $\left(\mathbf{H}_{k(\alpha)}^{+}\right)_{i j}$ denotes the joint probability that the queue length is $k$ and the UMC phase is $j$ at (3) of Figure 3.1 given that the UMC phase is $i$ at (1),

(iii) $\left(\mathbf{H}_{k}\right)_{i j}$ denotes the probability that $k$ customers arrive during the setup time and the UMC phase is $j$ at (3) under the condition that the UMC phase is $i$ at (2).

We define the matrix GFs of the above probability matrices as follows:

$$
\mathbf{H}_{\alpha}^{-}(z)=\sum_{k=\alpha}^{\infty} \mathbf{H}_{k(\alpha)}^{-} z^{k}, \quad \mathbf{H}_{\alpha}^{+}(z)=\sum_{k=\alpha}^{\infty} \mathbf{H}_{k(\alpha)}^{+} z^{k}
$$

Then, we have the following theorem.

Theorem 4.4. There exist

$$
\begin{aligned}
\mathbf{H}_{k(\alpha)}^{-} & =\mathbf{Q}_{k}^{\alpha}, \\
\mathbf{H}_{\alpha}^{-}(z) & =\left[\sum_{n=0}^{\alpha-1} \mathbf{D}_{n}^{*}\left(\mathbf{I}-\mathbf{D}_{0}\right)^{-1} z^{n}\right]\left[\mathbf{D}(z)-\mathbf{D}_{0}\right]-\sum_{n=1}^{\alpha-1} \mathbf{D}_{n}^{*} z^{n}, \\
\mathbf{H}_{k(\alpha)}^{+} & =\sum_{i=\alpha}^{k} \mathbf{H}_{i(\alpha)}^{-} \mathbf{H}_{k-i}, \\
\mathbf{H}_{\alpha}^{+}(z) & =\mathbf{H}_{\alpha}^{-}(z) \mathbf{H}(z) \\
\mathbf{\Psi}_{k}^{\mathrm{sb}} & =\sum_{i=\alpha}^{k} \mathbf{H}_{i(\alpha)}^{+} \mathbf{D}_{k-i}^{*} .
\end{aligned}
$$


Proof. Equations (4.14a) and (4.14b) can be obtained by using $\alpha$ in place of $N$ in (4.12a) and (4.12b). Equations (4.14c) and (4.14d) are obvious. Equation (4.14e) can be obtained first by conditioning on the queue length at the end of the setup period and then by applying (4.10).

So far, we have obtained all the quantities that we need in (4.9) except $\boldsymbol{\kappa}$, which is the stationary phase probability vector at (1). Let $\mathbf{K}$ be the phase transition probability matrix between (1) and (5). Then, $\boldsymbol{\kappa}$ can be computed from (see Lucantoni [25])

$$
\begin{gathered}
\boldsymbol{\kappa} \mathbf{K}=\boldsymbol{\kappa}, \quad \boldsymbol{\kappa} \mathbf{e}=1, \\
\mathbf{K}=\left.\mathbf{K}(z)\right|_{z=1},
\end{gathered}
$$

where $\mathbf{K}(z)$ is the matrix GF of the number of customers that are served between (1) and (5). To obtain $\mathbf{K}(z)$, we need the information concerning the queue length at (4). Let $\left(\mathbf{Q}_{k}^{(\alpha, N)}\right)_{i j}$ be the probability that the queue length is $k$ and the UMC is in phase $j$ at (4) under the condition that the UMC is in phase $i$ at (1) $(k \geq N)$. We define the matrix GF $\mathbf{Q}_{(\alpha, N)}(z)=\sum_{k=N}^{\infty} \mathbf{Q}_{k}^{(\alpha, N)} z^{k}$. Then, we have the following.

Theorem 4.5 .

$$
\begin{gathered}
\mathbf{Q}_{n}^{(\alpha, N)}=\mathbf{H}_{n(\alpha)}^{+}+\sum_{j=\alpha}^{N-1} \mathbf{H}_{j(\alpha)}^{+} \mathbf{Q}_{n-j}^{N-j} \quad(n \geq N), \\
\mathbf{Q}_{(\alpha, N)}(z)=\mathbf{H}_{\alpha}^{+}(z)+\sum_{j=\alpha}^{N-1} \Psi_{j}^{\mathrm{sb}} z^{j}\left\{\left(\mathbf{I}-\mathbf{D}_{0}\right)^{-1}\left[\mathbf{D}(z)-\mathbf{D}_{0}\right]-\mathbf{I}\right\} .
\end{gathered}
$$

Proof. Equation (4.17a) can be obtained by conditioning on the queue length at the end of the setup period. Multiplying (4.17a) by $z^{n}$ and summing over $n$, we get

$$
\begin{aligned}
\mathbf{Q}_{(\alpha, N)}(z) & =\sum_{n=N}^{\infty} \mathbf{H}_{n(\alpha)}^{+} z^{n}+\sum_{n=N}^{\infty}\left[\sum_{j=\alpha}^{N-1} \mathbf{H}_{j(\alpha)}^{+} \mathbf{Q}_{n-j}^{N-j}\right] z^{n} \\
& =\mathbf{H}_{\alpha}^{+}(z)-\sum_{n=\alpha}^{N-1} \mathbf{H}_{n(\alpha)}^{+} z^{n}+\sum_{j=\alpha}^{N-1} \mathbf{H}_{j(\alpha)}^{+} z^{j} \sum_{n=N}^{\infty} \mathbf{Q}_{n-j}^{N-j} z^{n-j} \\
& =\mathbf{H}_{\alpha}^{+}(z)+\sum_{j=\alpha}^{N-1} \mathbf{H}_{j(\alpha)}^{+} z^{j}\left[\sum_{n=N-j}^{\infty} \mathbf{Q}_{n}^{N-j} z^{n}-\mathbf{I}\right] .
\end{aligned}
$$

From the identity $\sum_{n=N-j}^{\infty} \mathbf{Q}_{n}^{N-j} z^{n}=\mathbf{Q}_{N-j}(z)$, and using (4.12b) and (4.14e), we get the second term of the last equality as 


$$
\begin{aligned}
\sum_{j=\alpha}^{N-1} \mathbf{H}_{j(\alpha)}^{+} z^{j}\left[\sum_{n=N-j}^{\infty} \mathbf{Q}_{n}^{N-j} z^{n}-\mathbf{I}\right] & \\
= & \sum_{j=\alpha}^{N-1} \mathbf{H}_{j(\alpha)}^{+} z^{j}\left[\left\{\sum_{n=0}^{N-j-1} \mathbf{D}_{n}^{*}\left(\mathbf{I}-\mathbf{D}_{0}\right)^{-1} z^{n}\right\}\left[\mathbf{D}(z)-\mathbf{D}_{0}\right]-\sum_{n=1}^{N-j-1} \mathbf{D}_{n}^{*} z^{n}-\mathbf{I}\right] \\
= & \sum_{j=\alpha}^{N-1} \mathbf{H}_{j(\alpha)}^{+} z^{j} \sum_{n=0}^{N-j-1} \mathbf{D}_{n}^{*}\left(\mathbf{I}-\mathbf{D}_{0}\right)^{-1} z^{n}\left[\mathbf{D}(z)-\mathbf{D}_{0}\right]-\sum_{j=\alpha}^{N-1} \mathbf{H}_{j(\alpha)}^{+} z^{j} \sum_{n=0}^{N-j-1} \mathbf{D}_{n}^{*} z^{n} \\
= & \sum_{k=\alpha}^{N-1} \sum_{i=0}^{k} \mathbf{H}_{i(\alpha)}^{+} \mathbf{D}_{k-i}^{*}\left(\mathbf{I}-\mathbf{D}_{0}\right)^{-1} z^{k}\left[\mathbf{D}(z)-\mathbf{D}_{0}\right]-\sum_{k=\alpha}^{N-1} \sum_{i=0}^{k} \mathbf{H}_{i(\alpha)}^{+} \mathbf{D}_{k-i}^{*} z^{k} \\
= & \sum_{k=\alpha}^{N-1} \mathbf{\Psi}_{k}^{\mathrm{sb}} z^{k}\left[\left(\mathbf{I}-\mathbf{D}_{0}\right)^{-1}\left(\mathbf{D}(z)-\mathbf{D}_{0}\right)-\mathbf{I}\right],
\end{aligned}
$$

which proves $(4.17 \mathrm{~b})$.

Now, we have

$$
\begin{aligned}
\mathbf{K}(z)= & \left.\mathbf{Q}_{(\alpha, N)}(z)\right|_{z=\mathbf{G}(z)} \\
= & {\left[\sum_{n=0}^{\alpha-1} \mathbf{D}_{n}^{*}\left[\mathbf{I}-\mathbf{D}_{0}\right]^{-1}[\mathbf{G}(z)]^{n}\right]\left[\mathbf{D}(\mathbf{G}(z))-\mathbf{D}_{0}\right] \mathbf{H}(\mathbf{G}(z)) } \\
& -\sum_{n=1}^{\alpha-1} \mathbf{D}_{n}^{*}[\mathbf{G}(z)]^{n} \mathbf{H}(\mathbf{G}(z)) \\
& +\sum_{n=\alpha}^{N-1} \Psi_{n}^{\mathrm{sb}}[\mathbf{G}(z)]^{n}\left\{\left(\mathbf{I}-\mathbf{D}_{0}\right)^{-1}\left[\mathbf{D}(\mathbf{G}(z))-\mathbf{D}_{0}\right]-\mathbf{I}\right\} \\
\mathbf{K}=\left.\mathbf{K}(z)\right|_{z=1}=\left[\sum_{n=0}^{\alpha-1} \mathbf{D}_{n}^{*}\left(\mathbf{I}-\mathbf{D}_{0}\right)^{-1} \mathbf{G}^{n}\right]\left(\mathbf{D}(\mathbf{G})-\mathbf{D}_{0}\right) \mathbf{H}(\mathbf{G}) & \quad-\sum_{n=1}^{\alpha-1} \mathbf{D}_{n}^{*} \mathbf{G}^{n} \mathbf{H}(\mathbf{G})+\sum_{n=\alpha}^{N-1} \Psi_{n}^{\mathrm{sb}} \mathbf{G}^{n}\left[\left(\mathbf{I}-\mathbf{D}_{0}\right)^{-1}\left(\mathbf{D}(\mathbf{G})-\mathbf{D}_{0}\right)-\mathbf{I}\right]
\end{aligned}
$$

In (4.20a), (4.20b), $\mathrm{G}(z)$ is the matrix GF of the number of customers that are served during a fundamental period (see Neuts [29]) which is given by

$$
\mathbf{G}(z)=z \sum_{k=1}^{\infty} s_{k}[\mathbf{D}(\mathbf{G}(z))]^{k},
$$


and $\mathbf{G}$ is the phase transition matrix during the fundamental period, which can be obtained as follows:

$$
\mathbf{G}=\left.\mathbf{G}(z)\right|_{z=1}=\sum_{k=1}^{\infty} s_{k}[\mathbf{D}(\mathbf{G})]^{k}
$$

Also, we have $\mathbf{H}(\mathbf{G}(z))=\sum_{k=1}^{\infty} h_{k}[\mathbf{D}(\mathbf{G}(z))]^{k}$ and $\mathbf{H}(\mathbf{G})=\left.\mathbf{H}(\mathbf{G}(z))\right|_{z=1}$.

Now, by computing $\boldsymbol{\kappa}$ from (4.15) with $\mathrm{K}$ obtained in (4.20b), we can determine all the quantities that we need for complete $\mathbf{p}_{\text {idle }}(z)$ in (4.9).

\section{Mean queue length}

The mean queue length $L$ can be obtained by following the standard procedure of Lucantoni [25]. We will skip the detailed derivation and will list only the results here. For notational conveniences, we will use $\mathbf{E}^{(n)}$ to denote $\left.\left(d^{n} / d z^{n}\right) \mathbf{E}(z)\right|_{z=1}$, and $\mathbf{p}_{\text {idle }}$ to denote $\left.\mathbf{p}_{\text {idle }}(z)\right|_{z=1}$ :

$$
L=\mathbf{Y}^{(1)} \mathbf{e}=\frac{1}{\lambda} \mathbf{U}^{(1)} \mathbf{e}-\frac{1}{2 \lambda} \pi \mathbf{D}^{(2)} \mathbf{e}-\frac{1}{\lambda}\left(\mathbf{U}-\pi \mathbf{D}^{(1)}\right)(\mathbf{D}-\mathbf{I}+\mathbf{e} \boldsymbol{\pi})^{-1} \mathbf{D}^{(1)} \mathbf{e},
$$

where

$$
\begin{gathered}
\mathbf{U}=\lambda \boldsymbol{\pi}(\mathbf{I}-\mathbf{A}+\mathbf{e} \boldsymbol{\pi})^{-1}+\mathbf{p}_{\text {idle }} \cdot(1-\rho)(\mathbf{D}-\mathbf{I}) \mathbf{A}(\mathbf{I}-\mathbf{A}+\mathbf{e} \boldsymbol{\pi})^{-1}, \\
\mathbf{U}^{-1} \mathbf{e}=\frac{1}{1-\rho}\left(F_{1}+F_{2}+F_{3}+F_{4}+F_{5}\right) .
\end{gathered}
$$

In $(5.2 \mathrm{~b}), F_{1}, F_{2}, F_{3}, F_{4}$, and $F_{5}$ are given, with $\delta=\boldsymbol{\kappa}(1-\rho) / E(I)$, by

$$
\begin{aligned}
F_{1}=\frac{\delta}{2}[ & 2 \sum_{k=1}^{\alpha-1} k \mathbf{D}_{k}^{*}\left(\mathbf{I}-\mathbf{D}_{0}\right)^{-1} \mathbf{D}^{(1)}+\sum_{k=0}^{\alpha-1} \mathbf{D}_{k}^{*}\left(\mathbf{I}-\mathbf{D}_{0}\right)^{-1} \mathbf{D}^{(2)} \\
& +2 \sum_{k=1}^{\alpha-1} k \mathbf{D}_{k}^{*}\left(\mathbf{I}-\mathbf{D}_{0}\right)^{-1} \mathbf{D}^{(1)} \mathbf{H}^{(1)}-2 \sum_{k=1}^{\alpha-1} k \mathbf{D}_{k}^{*} \mathbf{H}^{(1)} \\
& +2 \sum_{k=1}^{\alpha-1} k \mathbf{D}_{k}^{*}\left(\mathbf{I}-\mathbf{D}_{0}\right)^{-1}\left(\mathbf{D}-\mathbf{D}_{0}\right) \mathbf{H}^{(1)} \\
& +\sum_{k=0}^{\alpha-1} \mathbf{D}_{k}^{*}\left(\mathbf{I}-\mathbf{D}_{0}\right)^{-1}\left(\mathbf{D}-\mathbf{D}_{0}\right) \mathbf{H}^{(2)}-\sum_{k=1}^{\alpha-1} \mathbf{D}_{k}^{*} \mathbf{H}^{(2)} \\
& \left.+2 \sum_{k=\alpha}^{N-1} k \Psi_{k}^{\mathrm{sb}}\left(\mathbf{I}-\mathbf{D}_{0}\right)^{-1} \mathbf{D}^{(1)}+\sum_{k=\alpha}^{N-1} \Psi_{k}^{\mathrm{sb}}\left(\mathbf{I}-\mathbf{D}_{0}\right)^{-1} \mathbf{D}^{(2)}\right] \mathbf{e},
\end{aligned}
$$


Ho Woo Lee et al. 129

$$
\begin{aligned}
F_{2}=\delta[ & \sum_{k=1}^{\alpha-1} k \mathbf{D}_{k}^{*}\left(\mathbf{I}-\mathbf{D}_{0}\right)^{-1}(\mathbf{D}-\mathbf{I})+\sum_{k=0}^{\alpha-1} \mathbf{D}_{k}^{*}\left(\mathbf{I}-\mathbf{D}_{0}\right)^{-1} \mathbf{D}^{(1)} \\
& +\sum_{k=1}^{\alpha-1} k \mathbf{D}_{k}^{*}\left(\mathbf{I}-\mathbf{D}_{0}\right)^{-1}\left(\mathbf{D}-\mathbf{D}_{0}\right)(\mathbf{H}-\mathbf{I}) \\
& +\sum_{k=0}^{\alpha-1} \mathbf{D}_{k}^{*}\left(\mathbf{I}-\mathbf{D}_{0}\right)^{-1} \mathbf{D}^{(1)}(\mathbf{H}-\mathbf{I})-\sum_{k=1}^{\alpha-1} k \mathbf{D}_{k}^{*}(\mathbf{H}-\mathbf{I}) \\
& +\sum_{k=0}^{\alpha-1} \mathbf{D}_{k}^{*}\left(\mathbf{I}-\mathbf{D}_{0}\right)^{-1}\left(\mathbf{D}-\mathbf{D}_{0}\right) \mathbf{H}^{(1)}-\sum_{k=1}^{\alpha-1} \mathbf{D}_{k}^{*} \mathbf{H}^{(1)} \\
& \left.+\sum_{k=\alpha}^{N-1} k \Psi_{k}^{\mathrm{sb}}\left(\mathbf{I}-\mathbf{D}_{0}\right)^{-1}(\mathbf{D}-\mathbf{I})+\sum_{k=\alpha}^{N-1} \mathbf{\Psi}_{k}^{\mathrm{sb}}\left(\mathbf{I}-\mathbf{D}_{0}\right)^{-1} \mathbf{D}^{(1)}\right] \mathbf{A}^{(1)} \mathbf{e},
\end{aligned}
$$

$$
\begin{aligned}
F_{3}=\frac{\delta}{2}[ & \sum_{k=0}^{\alpha-1} \mathbf{D}_{k}^{*}\left(\mathbf{I}-\mathbf{D}_{0}\right)^{-1}(\mathbf{D}-\mathbf{I})-\sum_{k=1}^{\alpha-1} \mathbf{D}_{k}^{*}(\mathbf{H}-\mathbf{I}) \\
& +\sum_{k=0}^{\alpha-1} \mathbf{D}_{k}^{*}\left(\mathbf{I}-\mathbf{D}_{0}\right)^{-1}\left(\mathbf{D}-\mathbf{D}_{0}\right)(\mathbf{H}-\mathbf{I}) \\
& \left.+\sum_{k=\alpha}^{N-1} \Psi_{k}^{\mathrm{sb}}\left(\mathbf{I}-\mathbf{D}_{0}\right)^{-1}(\mathbf{D}-\mathbf{I})\right] \mathbf{A}^{(2)} \mathbf{e}+\frac{1}{2} \mathbf{U} \mathbf{A}^{(2)} \mathbf{e},
\end{aligned}
$$$$
F_{4}=-\delta\left[\sum_{k=1}^{\alpha-1} k \mathbf{D}_{k}^{*}\left(\mathbf{I}-\mathbf{D}_{0}\right)^{-1}(\mathbf{D}-\mathbf{I})+\sum_{k=0}^{\alpha-1} \mathbf{D}_{k}^{*}\left(\mathbf{I}-\mathbf{D}_{0}\right)^{-1} \mathbf{D}^{(1)}\right.
$$$$
+\sum_{k=1}^{\alpha-1} k \mathbf{D}_{k}^{*}\left(\mathbf{I}-\mathbf{D}_{0}\right)^{-1}\left(\mathbf{D}-\mathbf{D}_{0}\right)(\mathbf{H}-\mathbf{I})
$$$$
+\sum_{k=0}^{\alpha-1} \mathbf{D}_{k}^{*}\left(\mathbf{I}-\mathbf{D}_{0}\right)^{-1} \mathbf{D}^{(1)}(\mathbf{H}-\mathbf{I})-\sum_{k=1}^{\alpha-1} k \mathbf{D}_{k}^{*}(\mathbf{H}-\mathbf{I})
$$$$
+\sum_{k=0}^{\alpha-1} \mathbf{D}_{k}^{*}\left(\mathbf{I}-\mathbf{D}_{0}\right)^{-1}\left(\mathbf{D}-\mathbf{D}_{0}\right) \mathbf{H}^{(1)}-\sum_{k=1}^{\alpha-1} \mathbf{D}_{k}^{*} \mathbf{H}^{(1)}
$$$$
\left.+\sum_{k=\alpha}^{N-1} k \Psi_{k}^{\mathrm{sb}}\left(\mathbf{I}-\mathbf{D}_{0}\right)^{-1}(\mathbf{D}-\mathbf{I})+\sum_{k=\alpha}^{N-1} \boldsymbol{\Psi}_{k}^{\mathrm{sb}}\left(\mathbf{I}-\mathbf{D}_{0}\right)^{-1} \mathbf{D}^{(1)}\right]
$$$$
\times \mathbf{A}(\mathbf{I}-\mathbf{A}+\mathbf{e} \boldsymbol{\pi})^{-1}\left(\mathbf{I}-\mathbf{A}^{(1)}\right) \mathbf{e},
$$

$$
\begin{aligned}
F_{5}=-\delta & {\left[\sum_{k=0}^{\alpha-1} \mathbf{D}_{k}^{*}\left(\mathbf{I}-\mathbf{D}_{0}\right)^{-1}(\mathbf{D}-\mathbf{I})-\sum_{k=1}^{\alpha-1} \mathbf{D}_{k}^{*}(\mathbf{H}-\mathbf{I})\right.} \\
& +\sum_{k=0}^{\alpha-1} \mathbf{D}_{k}^{*}\left(\mathbf{I}-\mathbf{D}_{0}\right)^{-1}\left(\mathbf{D}-\mathbf{D}_{0}\right)(\mathbf{H}-\mathbf{I})
\end{aligned}
$$




$$
\begin{aligned}
& \left.+\sum_{k=\alpha}^{N-1} \Psi_{k}^{\mathrm{sb}}\left(\mathbf{I}-\mathbf{D}_{0}\right)^{-1}(\mathbf{D}-\mathbf{I})\right] \mathbf{A}^{(1)}(\mathbf{I}-\mathbf{A}+\mathbf{e} \boldsymbol{\pi})^{-1}\left(\mathbf{I}-\mathbf{A}^{(1)}\right) \mathbf{e} \\
& +\mathbf{U}\left(\mathbf{I}-\mathbf{A}^{(1)}\right)(\mathbf{I}-\mathbf{A}+\mathbf{e} \boldsymbol{\pi})^{-1}\left(\mathbf{I}-\mathbf{A}^{(1)}\right) \mathbf{e} .
\end{aligned}
$$

\section{Acknowledgment}

This research was supported by KOSEF through the Statistical Research Center for Complex Systems at Seoul National University.

\section{References}

[1] A. S. Alfa and M. F. Neuts, Modelling vehicular traffic using the discrete time Markovian arrival process, Transport. Sci. 29 (1995), no. 2, 109-117.

[2] C. Blondia and O. Casals, Statistical multiplexing of VBR sources: matrix-analytic approach, Performance Evaluation 16 (1992), 5-20.

[3] H. Bruneel and B. G. Kim, Discrete Time Models for Communication Systems Including ATM, Kluwer Academic Publishers, Massachusetts, 1993.

[4] S. H. Chang, N. K. Kim, K. C. Chae, H. W. Lee, and T. Takine, Factorization properties in the discrete-time BMAP/G/1 queue with generalized vacations, preprint, 2002.

[5] J. N. Daigle and S. C. Tang, The queue length distribution for multiserver discrete time queues with batch Markovian arrivals, Comm. Statist. Stochastic Models 8 (1992), no. 4, 665-683.

[6] I. Frigui, A. S. Alfa, and X. Xu, Algorithms for computing waiting time distributions under different queue disciplines for the D-BMAP/PH/1, Naval Res. Logist. 44 (1997), no. 6, 559-576.

[7] O. Hashida, Y. Takahashi, and S. Shimodawa, Switched batch Bernoulli process (SBBP) queue with application to statistical multiplexer performance, IEEE JSAC 9 (1991), no. 3, 394-401.

[8] M. Hersh and I. Brosh, The optimal strategy structure of an intermittently operated service channel, European J. Oper. Res. 5 (1980), no. 2, 133-141.

[9] M. Hofri, Queueng systems with a procrastinating server, Performance Evaluation Review 14 (1986), no. 1, 245-253.

[10] F. Ishizaki, T. Takine, Y. Takahashi, and T. Hasegawa, A generalized SBBP/G/1 queue and its applications, Performance Evaluation 21 (1994), 163-181.

[11] M. A. Johnson and S. Narayana, Descriptors of arrival-process burstiness with application to the discrete Markovian arrival process, Queueing Systems Theory Appl. 23 (1996), no. 1-4, 107130.

[12] K. H. Kang and C. H. Kim, Performance analysis of statistical multiplexing of heterogeneous discrete-time Markovian arrival processes in an ATM network, Computer Communications 20 (1997), 970-978.

[13] S. Kasahara, T. Takine, Y. Takahashi, and T. Hasegawa, Map/G/1 Queues Under N-Policy with and Without Vacations, Journal of the Operations Research Society of Japan 39 (1996), 188212.

[14] O. Kella, The threshold policy in the $M / G / 1$ queue with server vacations, Naval Res. Logist. 36 (1989), no. 1, 111-123.

[15] N. K. Kim, S. H. Chang, and K. C. Chae, On the relationships among queue lengths at arrival, departure, and random epochs in the discrete-time queue with D-BMAP arrivals, Oper. Res. Lett. 30 (2002), no. 1, 25-32.

[16] G. Latouche and V. Ramaswami, A unified stochastic model for the packet stream from periodic sources, Performance Evaluation 14 (1992), 103-121. 

Statistics and Applied Probability, Society for Industrial and Applied Mathematics (SIAM), Pennsylvania, 1999.

[18] H.-S. Lee and M. M. Srinivasan, Control policies for the $M^{X} / G / 1$ queueing system, Management Sci. 35 (1989), no. 6, 708-721.

[19] H. W. Lee, B. Y. Ahn, and N. I. Park, Decompositions of the queue length distributions in the MAP/G/1 queue under multiple and single vacations with N-Policy, Stoch. Models 17 (2001), no. 2, 157-190.

[20] H. W. Lee and J. O. Park, Optimal strategy in N-Policy production system with early set-up, J. Oper. Res. Soc. 48 (1997), 306-313.

[21] H. W. Lee, N. I. Park, and J. Jeon, Application of the factorization property to the analysis of production systems with a non-renewal input, bilevel threshold control, setup time and maintenance, Matrix-Analytic Methods: Theory and Applications (Proceeding of the International Conference on Matrix-Analytic Methods in Stochastic Models) (G. Latouche and P. Taylor, eds.), World Scientific Publishing, New Jersey, 2002, pp. 219-236.

[22] - A new approach to the queue length and waiting time of BMAP/G/1 queues, Comput. Oper. Res. 30 (2003), no. 13, 2021-2045.

[23] H. W. Lee, Y. J. Shin, and J. Jeon, A hybrid approach to analyze the discrete-time MAP/G/1 queues with control policies, Proceedings of the 7th Annual International Conference on Industrial Engineering (Busan, 2002), 2002, pp. 342-345.

[24] H. W. Lee, Y. J. Shin, E. Y. Lee, and K. C. Chae, Comparison of two approaches for discrete-time $M A P / G / 1$ queues, Proceedings of the 4th Asia-Pacific Conference on Industrial Engineering and Management Systems (Taipei, 2002), 2002, pp. 570-573.

[25] D. M. Lucantoni, New results on the single server queue with a batch Markovian arrival process, Comm. Statist. Stochastic Models 7 (1991), no. 1, 1-46.

[26] - The BMAP/G/1 Queue: A Tutorial, Models and Techniques for Performance Evaluation of Computer and Communications Systems (L. Donatiello and R. Nelson, eds.), Springer, New York, 1993, pp. 330-358.

[27] D. M. Lucantoni, K. S. Meier-Hellstern, and Marcel F. Neuts, A single-server queue with server vacations and a class of nonrenewal arrival processes, Adv. in Appl. Probab. 22 (1990), no. 3, 676-705.

[28] M. F. Neuts, A versatile Markovian point process, J. Appl. Probab. 16 (1979), no. 4, 764-779.

[29] Structured Stochastic Matrices of M/G/1 Type and Their Applications, Probability: Pure and Applied, vol. 5, Marcel Dekker, New York, 1989.

[30] D. Park and H. G. Perros, M-MMBP characterization of the departure process of an $M$ $M M B P / G e o / 1 / K$ queue, Proceedings of ITC 14, 1994, pp. 75-84.

[31] V. Ramaswami, The N/G/1 queue and its detailed analysis, Adv. in Appl. Probab. 12 (1980), no. $1,222-261$.

[32] V. Ramaswami and G. Latouche, Modeling packet arrivals from asynchronous input line, Proceedings of ITC 12, Torino, 1988.

[33] V. Ramaswami and J. L. Wang, A discrete time queueing analysis of ATM systems with phase-type distributed talk spurts traffic sources, IEEE International Conference on Communications, Texas, 1996, pp. 1093-1098.

[34] _ A discrete time queueing analysis of ATM systems with heterogeneous traffic sources, Proc. IEEE GLOBECOM. Vol 1 (London, UK, 1996), IEEE, New York, 1996, pp. 623-628.

[35] H. Takagi, Queueing Analysis: a Foundation of Performance Evaluation. Vol. 3. Discrete-Time Systems, North-Holland Publishing, Amsterdam, 1993.

[36] S. S. Wang and J. A. Silvester, A discrete-time performance model for integrated service ATM multiplexers, Proc. IEEE GLOBECOM (Houston, Tex, 1993), IEEE, New York, 1993, pp. 757761. 
132 Using factorization in analyzing D-BMAP/G/1 queues

[37] M. Yadin and P. Naor, Queueing system with removable service station, Opns. Res. Quarterly 14 (1963), 393-405.

Ho Woo Lee: Department of Systems Management Engineering, Sungkyunkwan University, Suwon Gyeonggi 440-746, Korea

E-mail address: hwlee@yurim.skku.ac.kr

Se Won Lee: Department of Systems Management Engineering, Sungkyunkwan University, Suwon Gyeonggi 440-746, Korea

Jongwoo Jeon: Department of Statistics, Seoul National University, Seoul 151-742, Korea

E-mail address: jwjeon@plaza.snu.ac.kr 


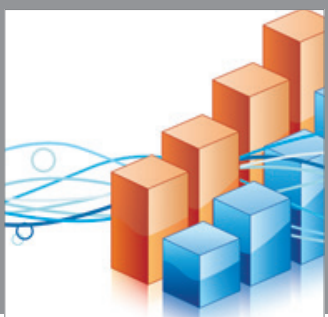

Advances in

Operations Research

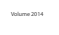

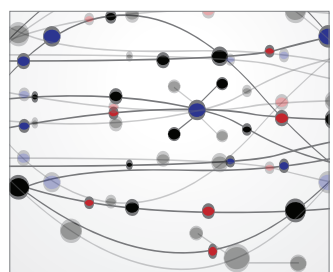

\section{The Scientific} World Journal
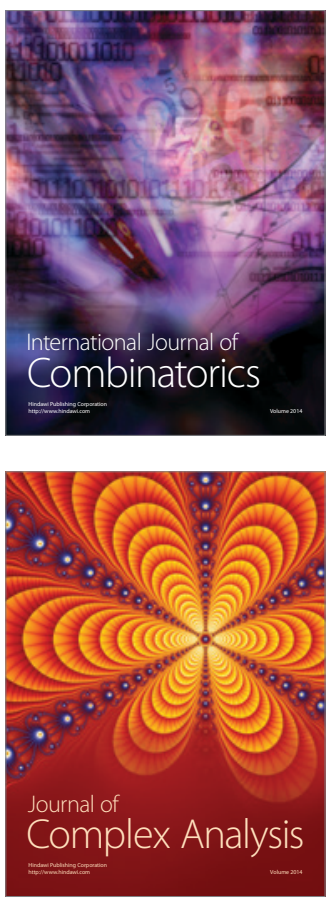

International Journal of

Mathematics and

Mathematical

Sciences
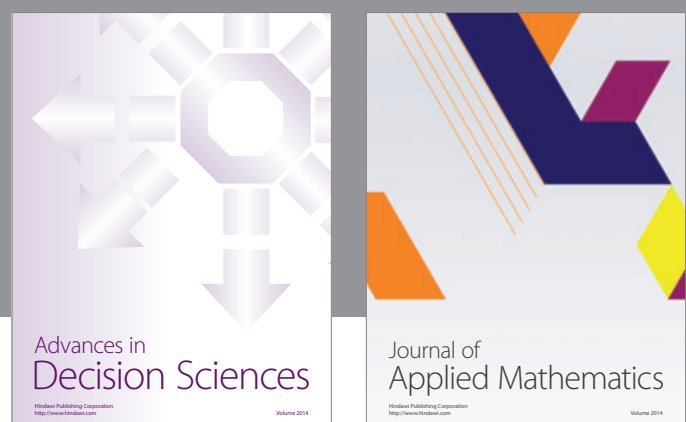

Journal of

Applied Mathematics
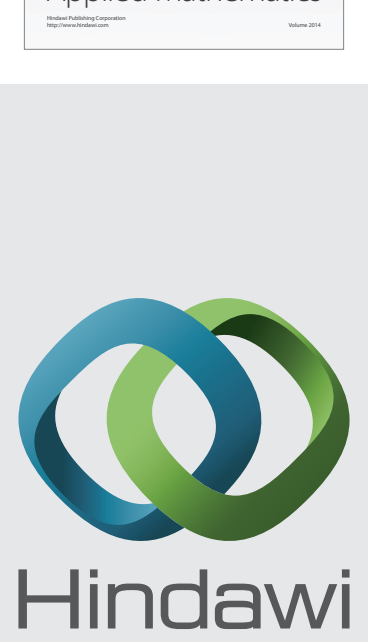

Submit your manuscripts at http://www.hindawi.com
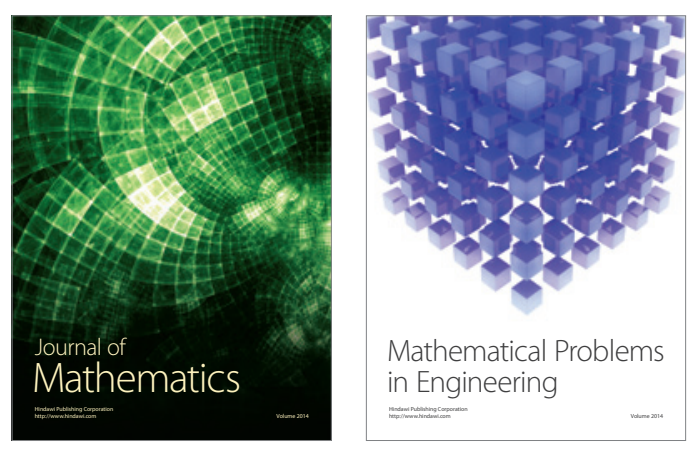

Mathematical Problems in Engineering
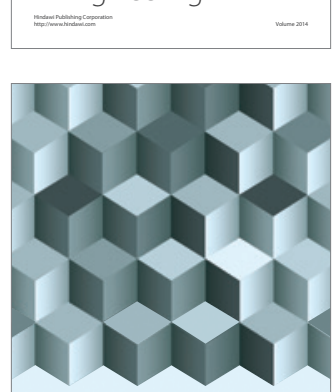

Journal of

Function Spaces
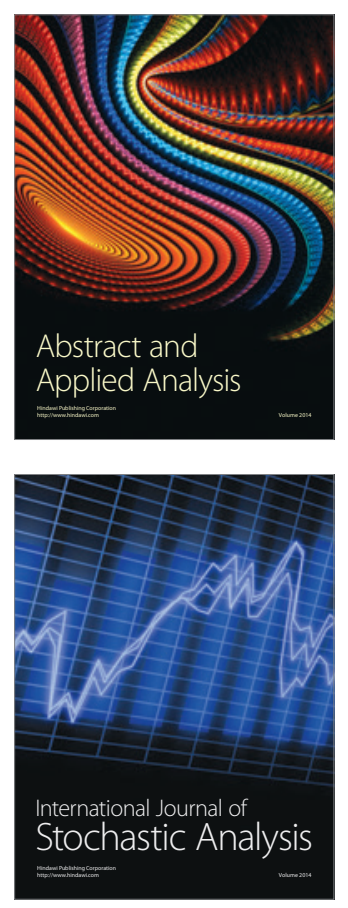

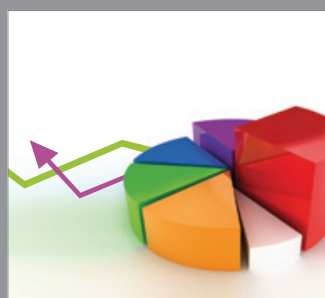

ournal of

Probability and Statistics

Promensencen
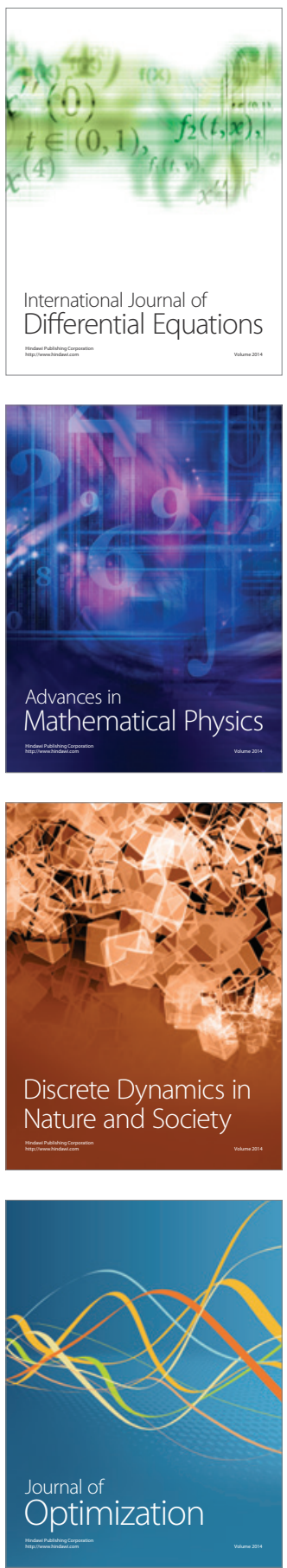